NIST Technical Note 2208

\title{
A Peg-in-Hole Test and Analysis Method for Exoskeleton Evaluation
}

\author{
Ann Virts \\ Roger Bostelman \\ Soocheol Yoon \\ Mili Shah \\ Ya-Shian Li-Baboud
}

This publication is available free of charge from: https://doi.org/10.6028/NIST.TN.2208

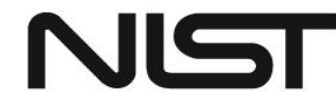




\title{
A Peg-in-Hole Test and Analysis Method for Exoskeleton Evaluation
}

\author{
Ann Virts \\ Roger Bostelman \\ Soocheol Yoon \\ Ya-Shian Li-Baboud \\ NIST \\ Mili Shah \\ The Cooper Union for the \\ Advancement of Science and Art
}

This publication is available free of charge from: https://doi.org/10.6028/NIST.TN.2208

March 2022

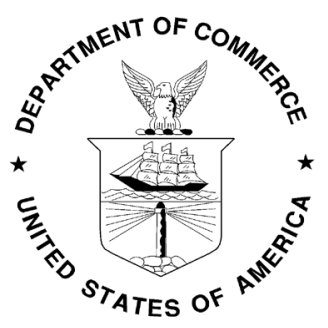

U.S. Department of Commerce

Gina M. Raimondo, Secretary

National Institute of Standards and Technology James K. Olthoff, Performing the Non-Exclusive Functions and Duties of the Under Secretary of Commerce for Standards and Technology \& Director, National Institute of Standards and Technology 
Certain commercial entities, equipment, or materials may be identified in this document in order to describe an experimental procedure or concept adequately. Such identification is not intended to imply recommendation or endorsement by the National Institute of Standards and Technology, nor is it intended to imply that the entities, materials, or equipment are necessarily the best available for the purpose.

National Institute of Standards and Technology Technical Note 2208

Natl. Inst. Stand. Technol. Tech. Note 2208, 31 pages (March 2022)

CODEN: NTNOEF

This publication is available free of charge from: https://doi.org/10.6028/NIST.TN.2208 


\begin{abstract}
Work-place MusculoSkeletal Disorders (WMSD) continue to be the most significant source of industrial workforce injuries. Exoskeletons are emerging as personal protective equipment (PPE) for sustained, repetitive, or intensive industrial tasks. This technical note describes and evaluates a proposed test method comprised of a novel testing apparatus, the Position and Load Test Apparatus for Exoskeletons (PoLoTAE), which was designed and developed at the National Institute of Standards and Technology (NIST), to simulate a wide variety of industrial tasks. The test method used commercially-available, low-cost and readilyadaptable sensors, building materials, and an initial set of qualitative and analytical methods to evaluate perceived user comfort, exoskeleton fit-for-task, and impact of exoskeleton on task productivity. The study includes analysis of simple subjective and quantitative measurement methods to obtain perceived comfort, effort, exoskeleton support, fit, task rate, and task completion times. Lastly, we include the limitations of this study and suggested improvements towards the development of test methods for the use of exoskeletons for industrial tasks, and towards providing more information for validation of models to understand the human-exoskeleton kinetic and kinematic interactions.
\end{abstract}

\title{
Key words
}

Exoskeleton test methods, standards, skeletal pose estimation, human-exoskeleton kinematics 


\section{Table of Contents}

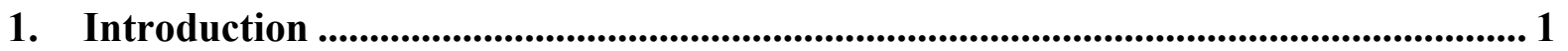

1.1. Towards Standard Test Methods for Industrial Exoskeletons.................................. 2

2. Exoskeleton Test Method Development ................................................................. 3

2.1. Human Subject Study ................................................................................... 3

2.3 Test Procedures ................................................................................... 4

3. Exoskeleton Evaluation Metrics............................................................................ 7

3.1. Exoskeleton Assessment .............................................................................. 7

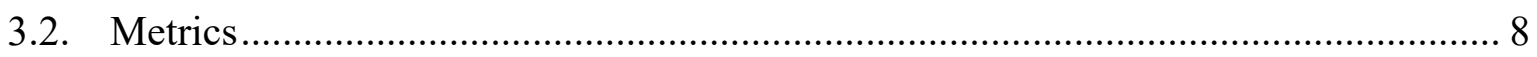

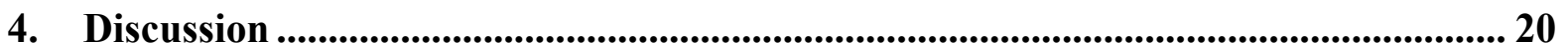

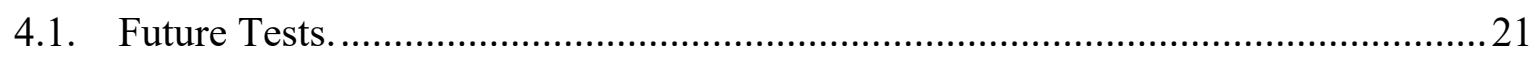

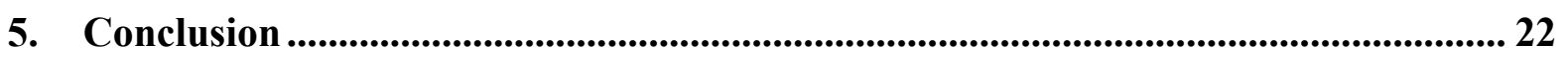

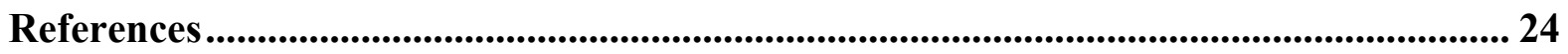




\section{Introduction}

Work-related Musculoskeletal Disorders (WMSDs) occur when occupational tasks cause over exertion and repetitive stress on muscles, tendons, and ligaments, which over time can lead to injury. Survey data from the Bureau of Labor Statistics indicated that the manufacturing industry had the third highest number of reported WMSD incidents in 2018 [1]. In 2001, the Institute of Medicine estimated the annual economic burden of WMSDs, due to productivity loss, compensation costs, and lost wages, was between $\$ 45$ and $\$ 54$ billion [2].

The ASTM F3323 - 20 Standard Terminology for Exoskeletons and Exosuits, defines an exoskeleton as a "wearable device that augments, enables, assists, or enhances physical activity through mechanical interaction with the body" [6]. Exoskeletons may include soft and rigid components. Exosuits provide a soft, conformant, and minimally obtrusive attire to support or augment human capabilities [7]. Exoskeletons and exosuits are being developed for industrial manufacturing applications to primarily support the user during potentially strenuous physical tasks, while also reducing fatigue and the risk of musculoskeletal injuries from repetitive tasks. The envisioned mechanical and power augmentation of exoskeletons is to support human capabilities and provide the potential benefit of retaining workers with experience and expertise. The exoskeletons in this study are designed to support the posture and loads during sustained, repetitive, or intensive industrial tasks. However, while exoskeletons reduce strain on the back and shoulders, the loads may be transferred to other parts of the body [14], and therefore the safety implications of the additional loads on the body should also be considered.

We visited an automobile manufacturing plant to observe a variety of tasks where the use of an exoskeleton can be used as personal protective equipment (PPE). For many tasks, including overhead drilling while working with the undercarriage of the vehicles or in a confined space working on the ceiling of the vehicle, the tasks were at a fixed height. The overhead tasks such as working on the undercarriage of a vehicle in a production factory, have a duration of ten minutes per vehicle, with workers completing three 2-hour shifts per day. Exoskeletons can support sustained and repetitive overhead work by distributing the musculoskeletal load from the shoulder and upper back and supporting the user and relieving strain caused by elevated arm positions and the weight of the tool.

As the industry begins to develop standard performance test methods for active and passive exoskeletons, quantifiable metrics to assess safety and ergonomic support are needed in order to understand how the exoskeleton can be applied to a specific task to prevent

musculoskeletal injuries. Subjective metrics, to assess user perception would also be useful in understanding the capabilities and limitations of an exoskeleton for a specific task. The metrics considered in this technical note and the experimental test methodologies developed are intended to inform standards development towards greater adoption of exoskeletons as a safety PPE in industry.

Prior studies have indicated that users would like to see improvements in the weight of the device, alignment with human anatomy and kinematics, and more fluid coordination in the 
human-robot interface to anticipate user motion and work to support the user's biomechanical load for a given set of tasks [8].

\subsection{Towards Standard Test Methods for Industrial Exoskeletons}

The challenges faced by evaluators of industrial exoskeletons and exosuits include the diversity of human anthropometric dimensions and preferences, the variety of industrial tasks and loads, and the range of operational environments. In evaluating the exoskeleton, the evaluation method should take into consideration the user population, the training required for both the evaluator and the user, the task environment, ergonomic and safety considerations, the user's measurements, and the biomechanical considerations involved in the physical activity. Research challenges include evaluation metrics and methods for exoskeletons in terms of safety, mobility, and usability [3].

There continues to be a need to identify metrics and test methods to evaluate the safety and performance of exoskeletons [4][5]. The metrics and test methods can inform standards efforts to assess exoskeleton safety, usability, and acceptability. In particular, the effects of the exoskeleton fit to the user and the user's stability while wearing the exoskeleton can have critical safety implications. International Organization for Standardization (ISO) 13482 Robots and robotic devices - Safety requirements for personal care robots defines safety standards for personal care robots with human-in-the-loop interactions, including mobile robots, robots used for transport, and exoskeletons [9]. However, industrial exoskeletons are subject to different functionality and safety concerns. Industrial exoskeletons are frequently used for a specific task with additional loads and forces. Tasks may require work in a confined space or with ergonomically sub-optimal angles.

The ASTM Committee F48 on Exoskeletons and Exosuits is developing standard test methods for exoskeletons in an effort to address safety, performance, and ergonomics [10]. To advance standards development and technology adoption efforts, the National Institute of Standards and Technology designed and developed a test platform, the Position and Load Test Apparatus for Exoskeletons (PoLoTAE), to simulate a variety of industrial tasks to evaluate exoskeletons designed for occupational use. Using the PoLoTAE, experimental industrial exoskeleton test methods, such as load handling [13], load alignment, force, and peg-in-hole, were designed and implemented. The development of a peg-in-hole test method was prioritized as hand tool use has led to a notable rate of WMSDs in the lower back and shoulder [22].

The objective of this study is to demonstrate and evaluate a peg-in-hole industrial task simulation for testing exoskeletons. The study is not a formal performance or safety evaluation of a specific exoskeleton. The study is intended to support the development of standard exoskeleton test methods to provide potential qualitative and quantitative metrics towards improved safety, effectiveness, and adoption of exoskeletons for industrial tasks.

This technical note describes our contribution to a proposed industrial exoskeleton performance measurement method for industrial peg-in-hole tasks. The test is based on (a) the use of a PoLoTAE test apparatus to be able to simulate industrial peg-in-hole tests; (b) an initial assessment of the test and measurement methodologies using low-cost sensors and 
building materials that are easily adaptable to industrial environments to evaluate exoskeleton tests; and (c) initial qualitative and analytical methods regarding the impact of an exoskeleton on user comfort, fit, and productivity. This technical note discusses the platform and performance measurement design, experiment, and analysis towards the development of an exoskeleton assessment method for peg-in-hole tasks.

\section{Exoskeleton Test Method Development}

\subsection{Human Subject Study}

The peg-in-hole industrial task tests were conducted over a period of two months from October 2018 to November 2018. This study was performed in accordance with the Institutional Review Board (IRB) at the National Institute of Standards and Technology (NIST) in the Exoskeleton test area. Nineteen self-declared healthy participants, ten female and nine male subjects, with no injuries at the time of the study gave written consent to participate in the study after being informed of the experimental test procedures. For this set of tasks, the subject's height and waist dimensions were estimated using waist size and visual inspection to fit the exoskeleton to the user. Five of the 19 participants had slight soreness or discomforts prior to the study. Empirical experience from fitting the exoskeleton indicated that the user's anthropometric dimensions can have a direct impact on the fit and comfort of the exoskeleton. The estimated height of the subjects ranged from $155 \mathrm{~cm}$ to $183 \mathrm{~cm}$. The estimated waist sizes ranged from approximately $64 \mathrm{~cm}$ to $91 \mathrm{~cm}$. The shoulder height was also measured for several subjects and ranged from $129.5 \mathrm{~cm}$ to $157.5 \mathrm{~cm}$. All participants were over the age of 18. Detailed tailor measurements and demographics data, such as the specific age of the subject, were not collected for this initial study, as the focus of the study was on the exoskeleton test methodology rather than the exoskeleton itself.

The test subjects were fitted to the same SuitX Max version 2 exoskeleton [26], which consisted of three parts: Leg-X, Back-X, and Shoulder-X. The team conducting the study was trained by the manufacturer on proper fitting, donning, doffing, and use of the exoskeleton. The exoskeleton size settings were adjusted to verify that the exoskeleton joints were properly aligned to key joints on the subject. For consistency in the fit of the exoskeleton, it was necessary to make the same study team member in charge of fitting the exoskeleton onto the test subjects. It was noted that in typical industrial settings, only a specific component, such as the Leg-X, would be worn, rather than the full suit. However, to focus on the test method development of all three exoskeleton components, rather than specifically evaluating the exoskeleton itself, the subjects were asked to wear all three components. The peg-in-hole test method was designed to use all three components, where the shoulder exoskeleton would support the overhead level peg-in-hole tasks, the back exoskeleton would support the middle level peg-in-hole tasks, and the leg exoskeleton would support the floor level peg-in-hole tasks.

The exoskeleton shoulder support provided assistive torque to reduce a user's muscle activation while completing repetitive or sustained overhead tasks [12]. For the set of experiments, the settings were configured to provide maximum peak torque amplitude 
support at the shoulder. The advantages of having the torque on the maximum setting were that it would provide the most support for the sustained overhead task at a specific angle, and the setting would be consistent across all subjects. However, the maximum torque also required additional exertion from the user when working at angles other than the intended angle, or range of angles supported by the exoskeleton. For some subjects, additional effort was needed to prevent the force of the exoskeleton from elevating the arms.

\subsection{Test Procedures}

Each subject performed a test without an exoskeleton (i.e., without wearing an exoskeleton) and a test with an exoskeleton (i.e., while wearing an exoskeleton). To reduce experimental confounding of assessment factors, the order of the tests was alternated between subjects. Nine of the participants began the tests without an exoskeleton, and 10 of the participants began the tests with an exoskeleton.

The task panels were fixed for all subjects regardless of their height. The rationale was based on our understanding of industrial manufacturing environments where the workers' positions relative to the tasks are typically fixed. The overhead task panel was fixed at $1.9 \mathrm{~m}$; the middle task panel was fixed at $1.5 \mathrm{~m}$; and the floor level task panel was fixed at $0.1 \mathrm{~m}$ high above the floor.

Fig. 1 shows the approximate test layout, including video camera locations and locations where still-frame photographs were taken relative to the subject and PoLoTAE test area. Fig. 2 shows a subject performing the test, overlaid with a skeletal pose estimation using OpenPose for assessing task completion times [27]. Each quad video consisted of two cameras trained on the subject from the rear left and rear right perspectives, a clock synchronized to the NIST time signal radio station, WWVB, in Fort Collins, $\mathrm{CO}$, air temperature and humidity, and a metabolic sensor display. 
Peg-In-Hole

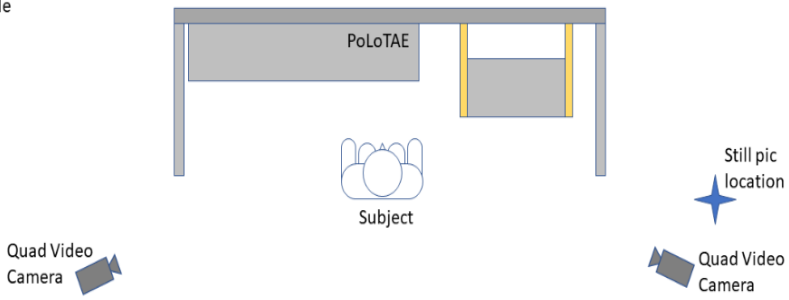

Exoskeleton

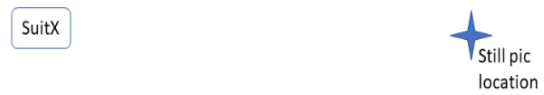

(a)

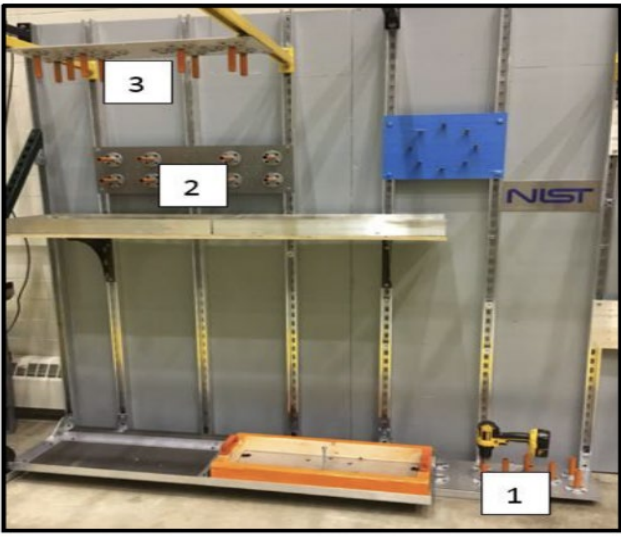

(b)

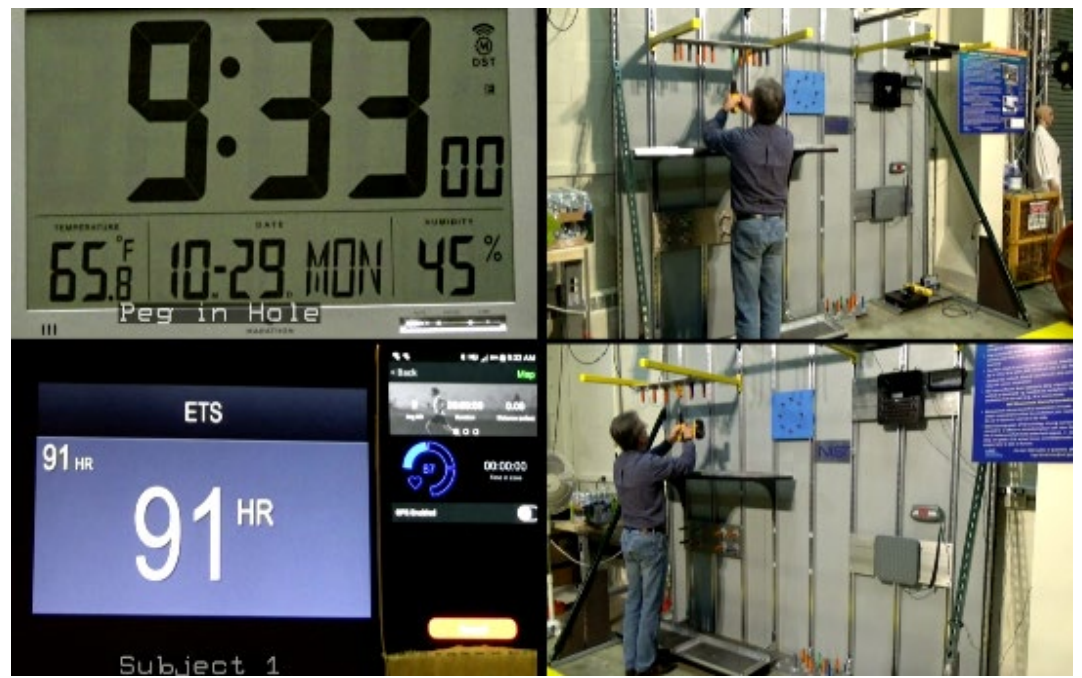

(c)

Fig. 1 (a) Test and measurement set up for peg-in-hole tasks with and without wearing the exoskeleton. (b) Peg-in-Hole task simulation using the PoLoTAE, where (1) simulated the floor task (pictured with the peg-in-hole tool), (2) simulated the wall task, and (3) simulated the overhead task. (c) Shows the display and the information from the quad video. 


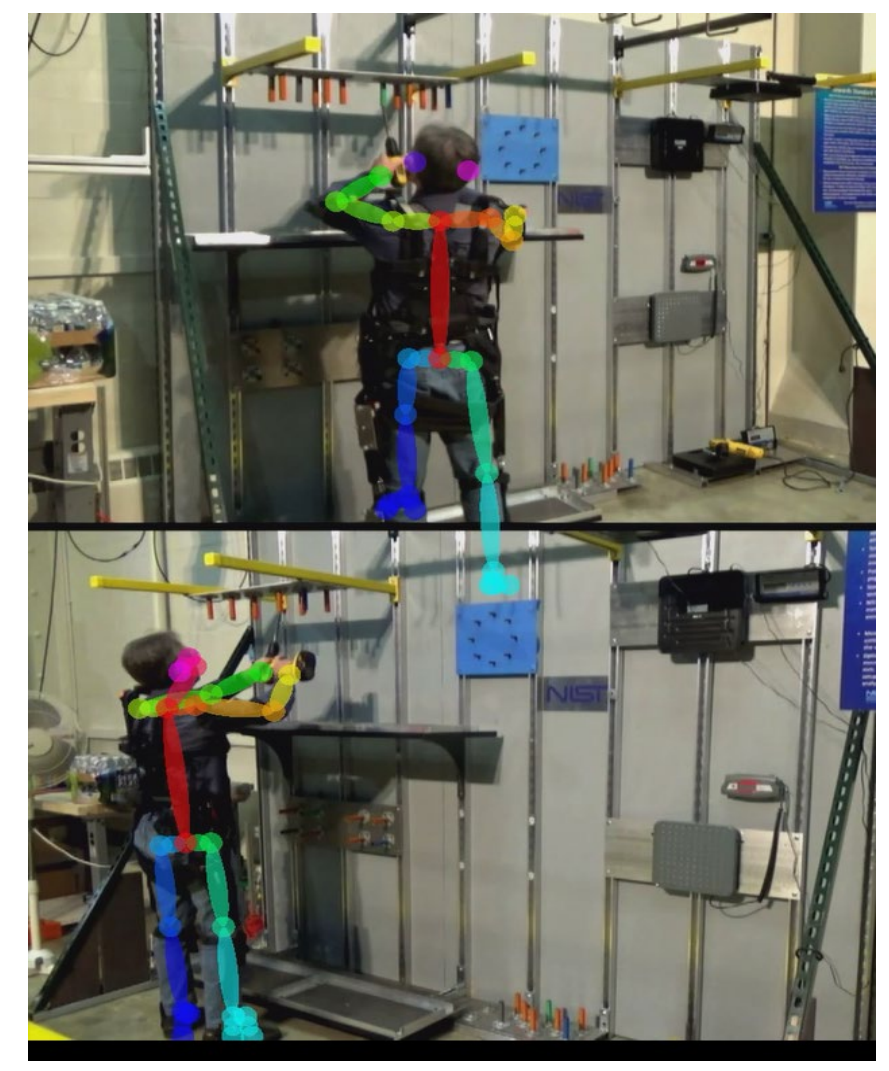

Fig. 2 Subject working with the NIST PoLoTAE exoskeleton test structure to assess task completion times for the peg-inhole tasks.

Prior to starting the test, each subject viewed a video of the task to understand the purpose of the exoskeleton study and an overview of the various industrial tasks the PoLoTAE was designed to test. A brief initial survey regarding any pains, soreness, or discomforts and a consent form were given. Upon consent, the subject proceeded to enter the test area.

The subject donned the exoskeleton depending on the alternating subject order of whether to start with the exoskeleton or without the exoskeleton. Subjects were instructed on a specific task order based on the color of the peg-in-hole tubes (blue, green, and gray), which they followed throughout their test and on all three (floor, wall, overhead) task plates (Figs. 1 and 2 ). The subject was asked to rest by sitting in a chair. Researchers attached two optical heart rate monitors to the subject. Heart rate was monitored, and when the subjects heart rate was stable based on visual assessment from the test operator, the resting heart rate was recorded on the test form. An electrical heart rate bar was also used to confirm the measurement. The subject was asked to stand at the start line holding the peg-in-hole tool (i.e., drill with blank drill bit) (Fig. 1 (b)), which weighed approximately $2.3 \mathrm{~kg}(5 \mathrm{lbs})$, and then began the trial after the data recording began.

The subject stepped forward to the PoLoTAE and inserted the peg-in-hole tool into the first colored tube followed by the next two colored tubes on the floor plate (Fig. 2). The subject 
then repeated the insertion process on the wall plate for the three colored tubes and on the overhead plate to complete one repetition of the trial. The subject repeated the order of the tasks for each plate 29 more repetitions to complete the trial, for a total of 270 peg-in-hole tool insertions.

Upon completion, the subject's heart rate was measured using the electrical heart rate monitor and recorded on the test form. The subject was asked to rest until their heart rate was within approximately 10 beats per second of their resting heart rate at the beginning of the first test. If the subject was wearing the exoskeleton during the trial, they were asked to stand next to a wall-mounted ruler to photograph their shoulder height. The subject was assisted with doffing or donning the exoskeleton for the second trial. After completing the second trial, the subject was asked to fill out a survey of questions regarding the test method, discomforts experienced during the test, and the exoskeleton in order to complete the test.

\section{Exoskeleton Evaluation Metrics}

\subsection{Exoskeleton Assessment}

When assessing exoskeletons, factors such as the exoskeleton's intended support profile [9][11], the task characteristics, the user's dimensions, fitness, and preferences must be considered. The amount of training and experience in working with the exoskeleton as well as the safety of the exoskeleton are also important considerations when designing a test method.

An exoskeleton's fit and support profile should be adapted on a task and user-specific basis. A study that evaluated a passive shoulder support exoskeleton demonstrated the ability to achieve optimal benefits and reduce safety risks by adapting the torque profile to the user and task preference [12]. In the development of a test method to assess the fit of lower-extremity exoskeletons and its impact on user stability, the results suggested proper alignment between the exoskeleton and the human joint improved user stability [13].

Current exoskeleton evaluations are often conducted in a laboratory environment. The assessment methods used in such evaluations have mainly been focused on the impact of the exoskeleton on musculoskeletal strain and fatigue. Several usability measures include qualitative perceived physical exertion, perceived comfort, and quantitative measures such as contact pressures and anthropometric fit [17].

Additional assessment techniques include the use of kinematic measures employing motion tracking systems, integrated potentiometers, and inertial measurement units (IMUs) to estimate joint angles and postures over time. Force plates and ground pressure contact sensors provide kinetic measures to assess biomechanical loading, gait, stability and balance. $[17][18][19]$.

Studies have also used physiological measures, such as heart rate, respiration rate, and perspiration levels to quantitatively assess full-body exertion levels. Electromyography (EMG) sensors have been applied to assess muscle activity in the primary muscles involved in a set of tasks, such as the lattimus dorsi, internal oblique, external oblique, rectus 
abdominis, erector spinae at the L3 vertebrae, and biceps femoris [15][20]. EMG can be a useful measure when evaluating lighter, repetitive work for all muscles involved in the tasks. EMG can be used to determine (a) whether a muscle was used, (b) the exertion level of the muscle, (c) force generation of the muscle when evaluated under specific conditions and the signals are calibrated and normalized, and (d) muscle fatigue when a standard submaximal isometric contraction is maintained, and the spectral components of the raw EMG signal are evaluated [21]. Biomechanical modeling, based on EMG data and a dynamic skeletal joint model along with customized active and passive muscle force algorithms, have also been used to understand and evaluate the biomechanical loading on human joints [22]. In particular, biomechanical modeling can determine whether the exoskeleton transfers the force from one area of the body to another.

Comfort measures include contact pressure to assess the compressive and shear forces between the exoskeleton and the human. Potential pressures exerted by the exoskeleton can cause discomfort or chafing [15] while completing a task. Prolonged pressure can create additional risks to the user.

Mobility measures for exoskeletons have also been assessed for specific tasks involving sitto-stand and stand-to-sit activities. Additionally, longitudinal studies on exoskeleton risk factors, including chronic cumulative trauma risks, will need to be considered [23].

Cognitive measures, such as, neurological sensors, response tracking, and eye tracking have been used [24] to determine whether additional cognitive load can impact the ability of the user to work safely, effectively, and synchronously with the exoskeleton [25].

\subsection{Metrics}

\subsubsection{Usability}

The effectiveness of human-exoskeleton interactions can be shaped by human factors, including perceived usability, frustration levels, and attitude, where a sincere effort to work in coordination with the exoskeleton can maximize the potential of the exoskeleton. This study relied on subjective survey questions to assess the usability of the exoskeleton as it pertains to the simulated industrial peg-in-hole task. As we gain insight from the initial qualitative metrics as to the challenges in the human-exoskeleton interface, the objective is to derive more quantitative measures for usability.

\subsubsection{Survey questions}

The survey included questions for evaluating the perceived usability of an exoskeleton and for evaluating the test method. The survey questions and scale are shown in Table 1.

Table 1 Survey questions for evaluating perceived usability of the exoskeleton for the simulated industrial peg-in-hole tasks.

\begin{tabular}{|c|c|c|}
\hline & Definition & Qualitative Measure \\
\hline Donning/Doffing & $\begin{array}{l}\text { Perceived ease of putting on and taking off the } \\
\text { exoskeleton. }\end{array}$ & Scale from 1 (no effort) to 5 (extreme effort) \\
\hline $\begin{array}{l}\text { Discomfort / Pains (prior/ } \\
\text { during / after) }\end{array}$ & $\begin{array}{l}\text { Perceived discomfort prior to and after using the } \\
\text { exoskeleton. }\end{array}$ & $\begin{array}{l}\text { Location of discomfort with scale from } 1 \\
\text { (slight), } 2 \text { (moderate), } 3 \text { (severe), and } 4 \\
\text { (extreme) }\end{array}$ \\
\hline Exoskeleton Comfort & Perceived comfort of the exoskeleton & $\begin{array}{l}\text { Scale from } 1 \text { (uncomfortable) to } 5 \text { (very } \\
\text { comfortable) }\end{array}$ \\
\hline Obstruction of Movement & Perceived obstruction in range of motion & $\begin{array}{l}\text { Scale from } 1 \text { (no obstruction) to } 5 \\
\text { (significant obstruction) }\end{array}$ \\
\hline
\end{tabular}




\begin{tabular}{lll}
\hline Ergonomic Support & Perceived ergonomic support provided by the exoskeleton & $\begin{array}{l}\text { Scale from } 1 \text { (strongly disagree) to } 5 \\
\text { (strongly agree) }\end{array}$ \\
Task Support & Perceived aid from the exoskeleton in completing the task & $\begin{array}{l}\text { Scale from } 1 \text { (strongly disagree) to } 5 \\
\text { (strongly agree) }\end{array}$ \\
\hline
\end{tabular}

\subsubsection{Physiological}

For this study, two optical heart rate monitors and an electrical heart rate sensor were used to measure the physical exertion. Heart rate was used to assess the physical intensity of the test method.

\subsubsection{Task metrics}

Productivity metrics, such as task completion time and task rate, can be considered pertinent to evaluating the impact of exoskeleton use on short-term user performance.

\subsubsection{Task perception metrics}

Finally, understanding the perceived task fidelity to practical uses and task exertion, from the perspective of an individual, can also be used to assess the exoskeleton test methodology and contribute to understanding the impact of the exoskeleton on the individual's task performance.

\subsubsection{Survey Questions}

Table 2 shows the survey questions pertaining to the test methodology and the results from the 19 subject responses. The objective of the survey was to inform potential test method improvements to understand the benefits and limitations of the exoskeleton with respect to an industrial manufacturing task that has shown to be a significant risk for WMSD for workers.

Table 2 Survey questions for evaluating the simulated exoskeleton peg-in-hole test method.

\begin{tabular}{|c|c|c|c|}
\hline & Definition & Qualitative Measure & Results [\%] \\
\hline Task fidelity & $\begin{array}{l}\text { Perceived the task properly } \\
\text { represents the real world. }\end{array}$ & $\begin{array}{l}\text { Scale from } 1 \text { (strongly disagree) to } 5 \\
\text { (strongly agree) }[\% \text { responded } \geq 3]\end{array}$ & $89 \%$ \\
\hline $\begin{array}{l}\text { Task used the } \\
\text { exoskeleton's capabilities }\end{array}$ & $\begin{array}{l}\text { Perceived the task used the } \\
\text { exoskeleton's intended capabilities. }\end{array}$ & $\begin{array}{l}\text { Scale from } 1 \text { (strongly disagree) to } 5 \\
\text { (strongly agree) }[\% \text { responded } \geq 3]\end{array}$ & $63 \%$ \\
\hline $\begin{array}{l}\text { Task used the } \\
\text { exoskeleton's limitations }\end{array}$ & $\begin{array}{l}\text { Perceived the task used the } \\
\text { exoskeleton's limitations. }\end{array}$ & $\begin{array}{l}\text { Scale from } 1 \text { (strongly disagree) to } 5 \\
\text { (strongly agree) }[\% \text { responded } \geq 3]\end{array}$ & $68 \%$ \\
\hline Easiest parts of the test & $\begin{array}{l}\text { Perceived ease in the different parts } \\
\text { of the task, the floor, overhead or } \\
\text { wall. }\end{array}$ & Open response & $52 \%$ (floor) \\
\hline $\begin{array}{l}\text { Most difficult parts of the } \\
\text { test }\end{array}$ & $\begin{array}{l}\text { Perceived difficulty in the different } \\
\text { parts of the task, the floor, overhead } \\
\text { or wall. }\end{array}$ & Open response & \\
\hline $\begin{array}{l}\text { Changes to the task to } \\
\text { make better use of the } \\
\text { exoskeleton }\end{array}$ & $\begin{array}{l}\text { Subject input in how they perceived } \\
\text { the exoskeleton can be used. }\end{array}$ & Open response & - \\
\hline $\begin{array}{l}\text { Maximum rep and } \\
\text { intensity (without } \\
\text { exoskeleton) }\end{array}$ & $\begin{array}{l}\text { Perceived physical intensity for the } \\
\text { user while working without the } \\
\text { exoskeleton. }\end{array}$ & $\begin{array}{l}\text { Scale from } 1 \text { (strongly disagree) to } 5 \\
\text { (strongly agree) [\% responded } \geq 3]\end{array}$ & $37 \%$ \\
\hline $\begin{array}{l}\text { Maximum rep and } \\
\text { intensity (with } \\
\text { exoskeleton) }\end{array}$ & $\begin{array}{l}\text { Perceived physical intensity for the } \\
\text { user while working with the } \\
\text { exoskeleton. }\end{array}$ & $\begin{array}{l}\text { Scale from } 1 \text { (strongly disagree) to } 5 \\
\text { (strongly agree) }[\% \text { responded } \geq 3]\end{array}$ & $11 \%$ \\
\hline Task Frustration & $\begin{array}{l}\text { Perceived frustration while } \\
\text { completing the task. }\end{array}$ & $\begin{array}{l}\text { Scale from } 1 \text { (strongly disagree) to } 5 \\
\text { (strongly agree) [\% responded } \geq 3]\end{array}$ & \\
\hline
\end{tabular}

\subsection{Analysis Methods}

The goal of the study was to evaluate the test method for its ability to assess the safety, usability, and productivity impact of industrial exoskeletons on industrial peg-in-hole tasks. 
Therefore, the analysis examined how the data collected can be used to assess the experimental procedures, the simulated task, the survey questions, and the metrics.

\subsubsection{Survey data}

\subsubsection{Exoskeleton assessment method}

Qualitative metrics regarding comfort, muscle soreness and fatigue, ergonomic support, and ease of donning the exoskeleton as well as an assessment of the test method were obtained from a set of survey questions administered using an online survey platform. Fig. 3 summarizes the survey questions on user assessment of wearing the exoskeleton. $79 \%$ felt it took "some" to "significant" effort to wear the exoskeleton. After performing the task, $68 \%$ of the users found the exoskeleton to be "neutral" to "comfortable" and "provided some ergonomic support during the task".

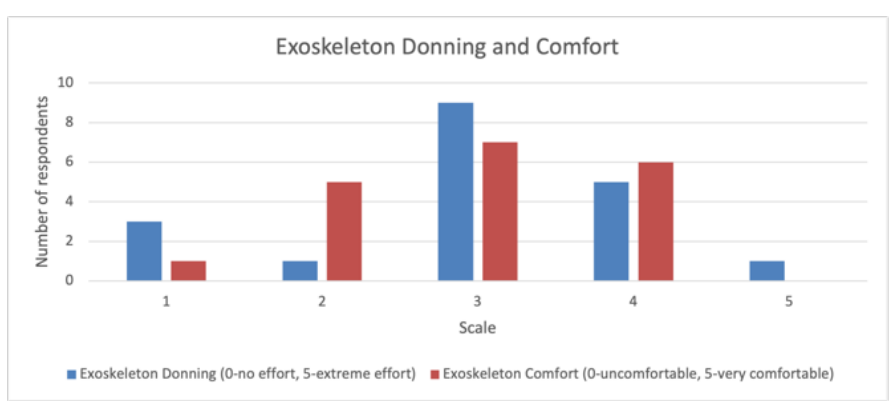

(a)

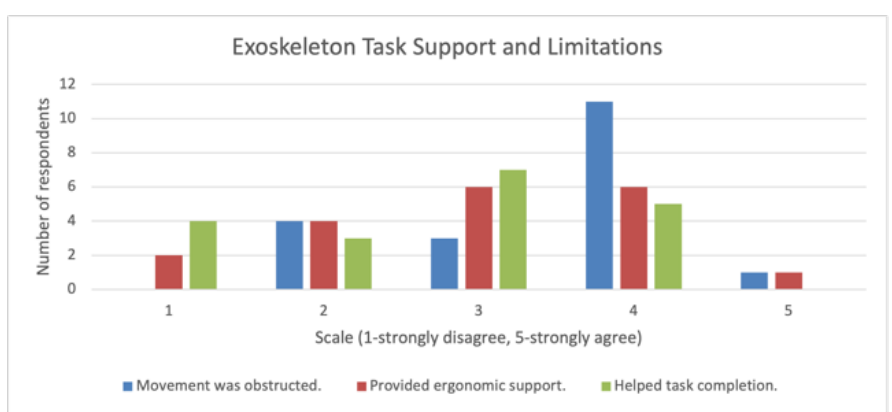

(b)

Fig. 3 Survey response distribution of qualitative assessment metrics for the user's perception of (a) the exoskeleton and (b) the exoskeleton relative to the peg-in-hole task.

For most of the users, the exoskeleton was best suited for the peg-in-hole task involving the lower pegs at the floor level. The users found the support of the leg and hips to be especially useful for prolonged tasks involving squatting and stooping, where the leg portion of the exoskeleton engaged to provide a chair-like support to the user.

\subsubsection{Task assessment method}

The survey also included several questions on whether the test provided adequate evaluation of the exoskeleton, and whether the test method can be improved. $89 \%$ of the respondents felt the peg-in-hole test method provided a "realistic simulation and provided information about the utility of the exoskeleton." To assess whether the test subjects felt the task was sufficiently strenuous to warrant the use of an exoskeleton, one of the survey questions asked 
whether the task was able to reach the test subjects' maximum load and repetition. $63 \%$ of the subjects felt the task reached their maximum intensity without the exoskeleton, compared to $89 \%$ when wearing the exoskeleton. The increase in perceived task intensity while wearing the exoskeleton indicated additional load of the exoskeleton along with the cognitive effort to work in coordination with the exoskeleton contributed to the task intensity. Fig. 4 shows the survey response distribution of the subjects' assessment of the test method. Additional input towards increasing task fidelity included focusing on one peg-in-hole plate level, such as the overhead plate, for a longer duration to allow the typical user to feel and appreciate the exoskeleton shoulder support as muscle soreness increases from a sustained and repetitive posture.

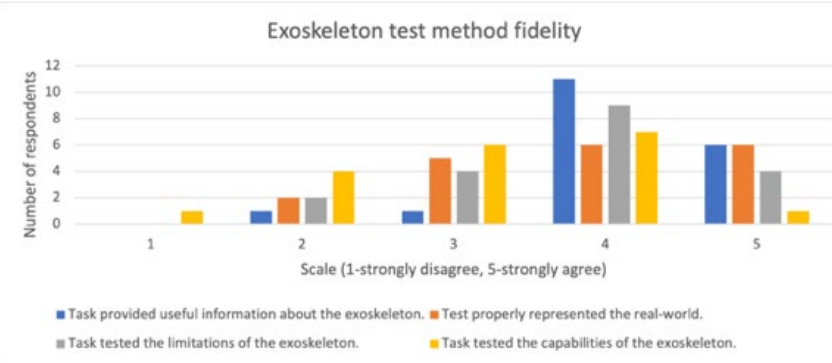

(a)

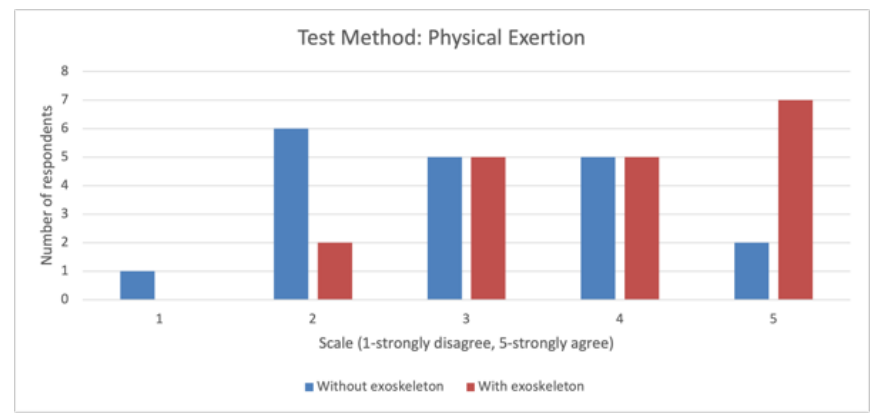

(b)

Fig. 4 Qualitative assessment metrics for the peg-in-hole exoskeleton test method (a) fidelity and (b) physical exertion.

\subsubsection{Fit and physical measurements}

The first step to assessing the fit was to visualize how the estimated anthropometric measurements or height and waist size compared to the exoskeleton settings. The study team was trained by the manufacturer in using and fitting the exoskeleton to a subject. For the fit analysis, the goal of the study was to determine how well the fit settings correlated with the physical estimates of height and waist size. The comparison is intended to understand which anthropometric measures are needed to provide a more precise metric for a specific exoskeleton size setting. Estimates from visual inspection were done rather than measurements, because the initial test method was intended to minimize the time and potential perceived intrusiveness for each subject if tailor measurements had been part of the test procedure. Additionally, as stated earlier, the intended goal was to focus on the test development process rather than actual exoskeleton evaluation. The goal of the fit analysis was to understand whether the height and waist size estimates were sufficient to accurately 
estimate the exoskeleton size setting or if more precise tailor measurements, and which types of tailor measurements, are needed for consistent exoskeleton fittings.

The subject's height appeared to be closely aligned with the exoskeleton spine setting. The tolerance between the fitted setting and the height of the subject appeared to be within $5 \mathrm{~cm}$. The settings for the leg exoskeleton also appear to be linear. The fitted hip sizes had a larger variation compared to the estimated waist. The variation may be due to the variability in how one may measure the waist and due to the differences in anthropometric waist measurement, depth or width, and the measurements needed to fit the exoskeleton's waist and hip settings.
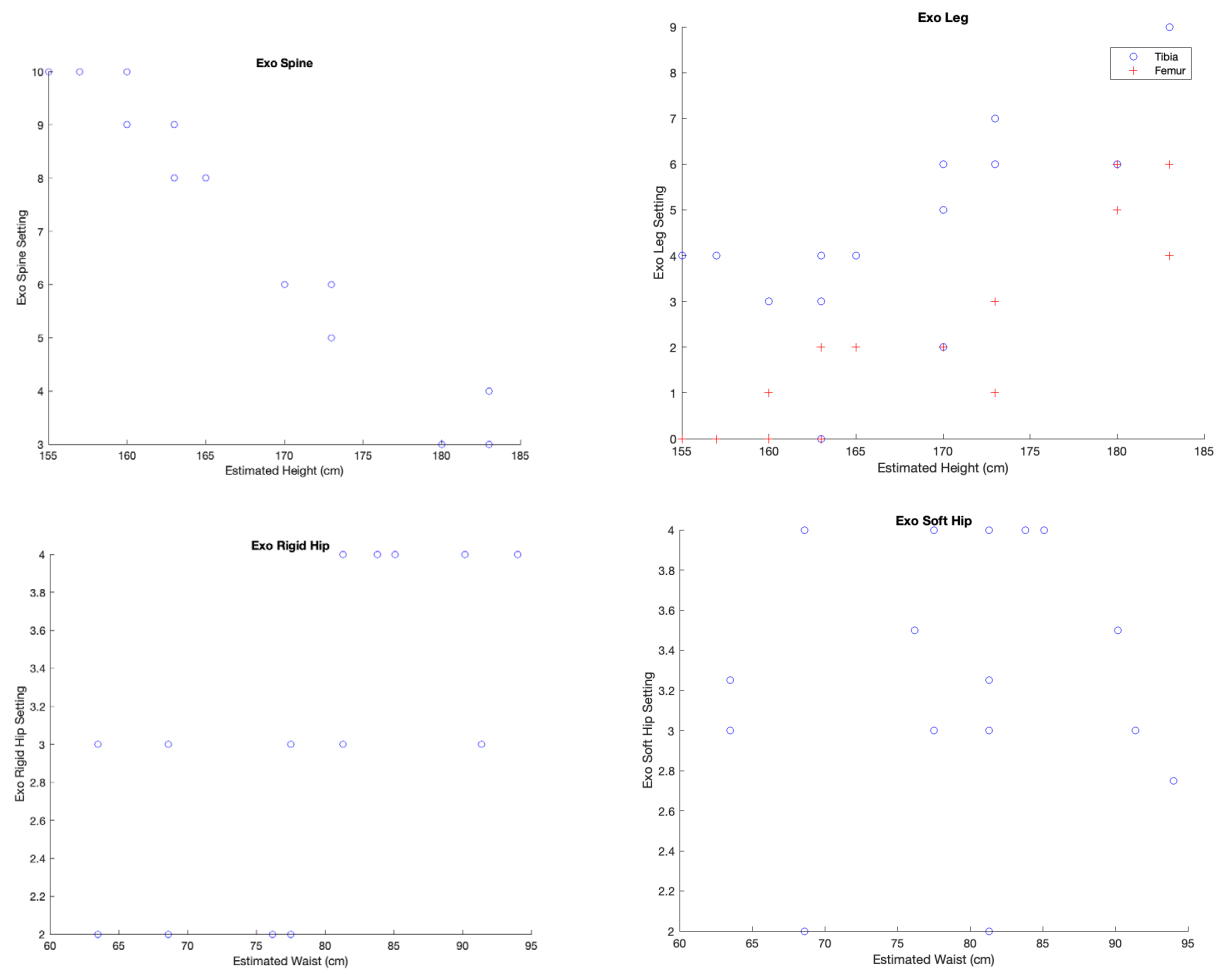

Fig. 5 Scatter plot of relationship between estimated weight and height of the subject and the exoskeleton spine, leg, and hip settings to show the anthropometric variation.

Upon visually assessing the trends between the estimated subject dimensions and the fitted settings, shown in Fig. 5, the relationship appears to be linear with respect to the subject's height and the spine, the femur, and the tibia settings. Note that the exoskeleton settings are specific to the exoskeleton used, and the trends would be different in other exoskeletons.

The Spearman correlation coefficient [28] was applied to assess whether there was a statistical relationship between the exoskeleton fit settings relative to the users' estimated measurements, as shown in Table 3. The Spearman correlation coefficient, $r$, was computed using Eq. 1 between the exoskeleton settings and the subjects' height and waist dimensions, where $\operatorname{cov}\left(r_{x}, r_{y}\right)$ is the covariance between the ranked subject dimension, $r_{x}$, and the ranked exoskeleton size setting, $r_{y}, \sigma_{r x}$ is the standard deviation of the ranked subjects' height or waist dimensions, and $\sigma_{r y}$ is the standard deviation of the ranked exoskeleton size setting for the spine, legs, and hips. The statistical significance was determined using critical value 
tables [28]. For 19 subjects, significance at the 0.75 quantile was 0.165 , at 0.90 was 0.309 , and 0.95 was 0.391 .

$$
r=\frac{\operatorname{cov}\left(r_{x}, r_{y}\right)}{\sigma_{r x} \sigma_{r y}} \quad \text { Eq. } 1
$$

The higher the correlation, the better the exoskeleton fit tolerances were to a specific height or waist size. The variation in settings for a given measurement can have several interpretations.

Table 3 Exoskeleton Fit Assessment Relative to Physical Height and Waist Dimensions

\begin{tabular}{lcc}
\hline \multicolumn{1}{c}{ Exoskeleton Setting } & Height & Waist \\
\hline Spine & $-0.97(\mathrm{p}$-value $=0.00)$ & N/A \\
Shoulder & $0.82(\mathrm{p}$-value $=0.01)$ & N/A \\
Shoulder-X & $0.84(\mathrm{p}$-value $=0.01)$ & N/A \\
Femur & $0.88(\mathrm{p}$-value $=0.00)$ & N/A \\
Tibia & $0.75(\mathrm{p}$-value $=0.01)$ & N/A \\
Arm & $-0.94(\mathrm{p}$-value $=0.00)$ & N/A \\
Waist & N/A & $0.60(\mathrm{p}$-value $=0.01)$ \\
\hline
\end{tabular}

The lower correlation between the exoskeleton's rigid waist setting is likely due to the need to find a better measurement to assess the setting for the rigid waist. For the exoskeleton used, the rigid waist frame sits closer to the hip, and therefore hip measurements may be a more accurate indicator of the exoskeleton's frame setting. Therefore, due to the variability in anthropometric dimensions, future tests may benefit from more specific tailor measurements, including shoulder width, hip circumference, hip depth, and leg measurements such as hip-to-knee and knee-to-heel. Higher correlations would indicate the most relevant anthropometric measurements in providing the sizing for a specific exoskeleton component. The goal of the subject size to exoskeleton settings analysis is to propose a systematic method to assess the anthropometric dimensions for exoskeleton fitting.

\subsubsection{Task Pain and Discomfort}

Before and after the test, each subject indicated whether they felt pain, discomfort, or soreness, and to indicate the severity of the discomfort and the specific location of the body. The number of subjects feeling discomfort, including soreness, rose from $26 \%$ to $42 \%$ after completion of the full set of tests. After the test, eight subjects indicated slight to severe discomfort in the front shoulder (deltoid) and biceps. Five subjects indicated slight to moderate discomfort in the knee and shin areas. Prior to the test, one subject indicated a slight discomfort in the front of the shoulder, and two subjects had indicated a slight discomfort in the knee and shin, respectively. The relative increase in discomfort may indicate either (1) the task was sufficiently intense to cause muscle strain in at least $42 \%$ of the subjects or (2) the exoskeleton and its settings were not fit for at least parts of the task in terms of the work angle relative to the subject. Specifically, the overhead task may not have been an optimal task angle for the configured exoskeleton under test, where the shoulder exoskeleton's torque was set at the maximum setting. The task angle is dependent on the 
user's height, and therefore was not consistent across all users. Some users found the exoskeleton provided support for the overhead tasks.

The users primarily indicated minimal discomfort with the lower-limb exoskeleton. Five of the 19 subjects had slight to moderate discomfort in the shin and knees due to pressure exerted by the exoskeleton in combination with the execution of the task.

About $25 \%$ of the participants indicated slight soreness or discomfort prior to the test. About $50 \%$ of the participants indicated soreness and discomfort during the test. The results subjectively indicated the task caused muscle strain in half of the users. When developing an industrial exoskeleton performance test method one consideration is the intensity of the activity for the intended population and deriving a threshold for the percentage of users. Further study using additional kinetic measurements and biomechanical modeling to derive force and load strain on the body is needed.

\subsubsection{Productivity Impact}

\subsubsection{Task Rate}

We allowed for the subject to become acclimated with the task first and used the latter half of the test, 15 repetitions, to determine the task rate. The task rate, TR (Eq. 2), was computed based on the number of pegs completed, $p$, over a measurement interval, $\Delta t$, using the following equation:

$$
\begin{gathered}
T R=\frac{p}{\Delta t} \quad \text { Eq. } 2 \\
\Delta T R=T R_{N E}-T R_{E} \quad \text { Eq. } 3
\end{gathered}
$$

$T R_{E}$ and $T R_{N E}$ are the task rate of the subject while wearing the exoskeleton and without the exoskeleton, respectively. The interval was determined using a 5-minute timer which began after the subject completed the $15^{\text {th }}$ repetition. After 5 minutes, the main test operator recorded the number of repetitions (and pegs) the subject completed within the time interval. The task rate differential was computed as $\Delta \mathrm{TR}$ (Eq. 3). Out of the 19 subjects, 17 subjects had a lower task rate while completing the tasks with the exoskeleton. One subject had a higher task rate, and one subject had the same task rate with and without the exoskeleton. Fig. 6 shows the relationship between the subject's height and the respective task rate with and without exoskeleton use. The highest dispersion between wearing and not wearing an exoskeleton appears to be subjects with a height at $170 \mathrm{~cm}$. At this height, the user may be impeded by the peak torque setting of the shoulder support. However, further study with more subjects, customized peak torque setting, and more consistency in subject training may be needed to determine whether benefits of exoskeletons are height dependent. One reason for the dependency on height is due to the difference in the angle of the task to the user. The angle of the task has been shown to be a factor in predicting muscle fatigue risk, and whether it exceeds the American Conference of Governmental Industrial Hygienists (ACGIH) threshold limit value (TLV) [29]. The ability to reduce muscle fatigue risk can be used to determine whether an exoskeleton is fit for a task. Statistically, however, there was no significant relationship between the height and task rate. However, there was an inverse relationship between height and whether the subject found the exoskeleton to be helpful in 
completing the task, as shown in Table 4. This may indicate that the exoskeleton better supported users that required more elevation and extension of the arm when completing the overhead peg-in-hole task.

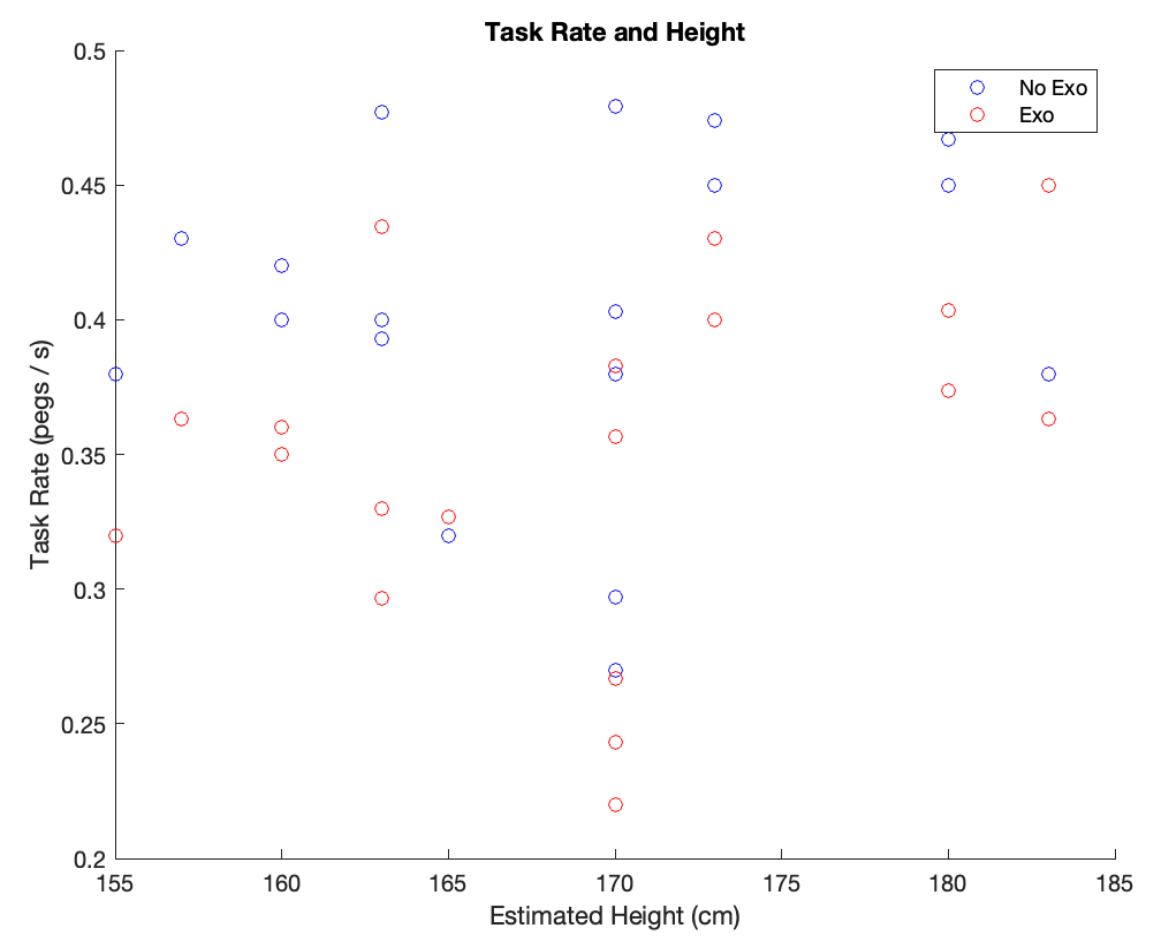

Fig. 6 Scatter plot of relationship between task rate and the subject's estimated height.

Table 4 Productivity impacts between the exoskeleton and the without exoskeleton trials for the simulated peg-in-hole test method.

\begin{tabular}{lcc}
\hline & Without Exoskeleton & Exoskeleton \\
\hline Average TR -Manual (pegs/s) [ $\sigma]$ & $0.41[0.06]$ & $0.35[0.06]$ \\
Rate decreased for last 15 repetitions (\%) & N/A & 15 \\
Average TR (overhead only) -Video (pegs/s) & 0.46 & 0.40 \\
Rate decreased for overhead pegs only (\%) & N/A & 11 \\
Overhead Task Completion Time - Intra-subject average (s) & $6.49[1.04]$ & $7.44[1.10]$ \\
Duration increased (\%) & & 13 \\
\hline
\end{tabular}

Table 5 Productivity and user perception metrics correlations to height and survey (correlation coefficient, $\boldsymbol{r}$ ).

\begin{tabular}{lcc}
\hline & Without Exoskeleton & Exoskeleton \\
\hline$T R(\boldsymbol{r})$ & 0.18 & 0.30 \\
$\Delta T R($ without exoskeleton-exoskeleton & N/A & 0.23 \\
Max intensity with exoskeleton $(\boldsymbol{r})$ & N/A & -0.31 \\
Exoskeleton (useful) $(\boldsymbol{r})$ & N/A & $\mathbf{- 0 . 4 1}$ \\
\hline
\end{tabular}

The analysis indicated the correlation between subject height and the user's perception on the usefulness of the exoskeleton was significant at the $95 \%$ confidence level for the sample size, $N=19$ [28]. The height of the user, which impacts the task and shoulder angle relationship, should be adjusted during the test to fit the exoskeleton's intended use profile. Furthermore, the shoulder torque adjustment may also need to fit the user preference based on their height to optimize the human-exoskeleton coordination in supporting the peg-in-hole tasks. In the free-form response, some users found the support in the overhead tasks and in 
the floor tasks helpful, while others responded that they felt they were working against the exoskeleton for the overhead tasks.

\subsubsection{Task Completion Time}

Video-based pose estimation was explored as a possible low-cost, quantitative method to determine task completion time of the overhead plate tasks. The pose estimation algorithm used a 25-point skeletal joint model as shown in Fig. 7. By following the $y$-coordinate on a point in the shoulder, elbow, or wrist, an algorithm was developed to determine the start of the task and the completion of the task. Each time the joint reached a peak height, the algorithm would count it as one repetition. The start of the task was determined based on the wrist height of the first repetition from the right and left camera perspectives, as shown in Fig. 8. The start of the task occurred when the wrist joint was higher than the shoulder. The end of the task was the last repetition, when the wrist went below the shoulder.

The task completion times were measured for the right and left arm respectively as shown in Fig. 9. The longer times were selected as final task completion times. Fig. 10 shows a task completion time of the selected subject over task repetitions. The figure shows that the subject's left arm task completion time increased when wearing an exoskeleton. This implies that wearing the exoskeleton changes the way tasks are performed. For several subjects, the pose estimation results were available only for one arm. Those occurred when the subjects performed tasks with one hand, with only one arm visible to the camera, and therefore the algorithm did not have sufficient information to estimate the pose of the occluded arm. Three subjects were excluded from the analysis as their pose estimation data were unavailable.

The overall mean task completion time without the exoskeleton was $6.49 \mathrm{~s}$ with a standard deviation of $1.04 \mathrm{~s}$. The overall mean task completion time with the exoskeleton was $7.435 \mathrm{~s}$ with a standard deviation of $1.10 \mathrm{~s}$. The overall mean task completion time increase was 15 $\%$. The largest change in task completion time was $1.80 \mathrm{~s}$ (or a $29.6 \%$ increase). Fig. 10 shows the inter-subject task completion time differences and changes.

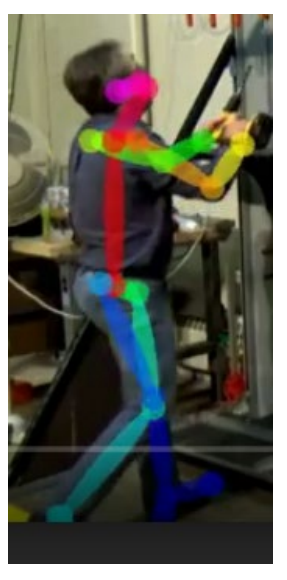

(a)

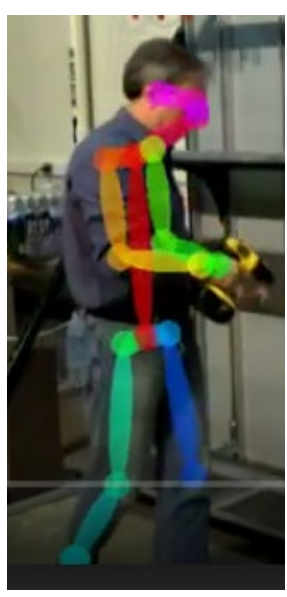

(b)

Fig. 7 Using video-based pose estimation techniques to estimate task completion time, where (a) is the Start position and (b) is the Stop position. 


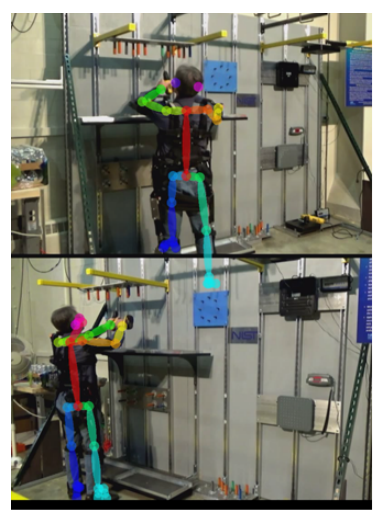

\section{From left (camera) view, left-arm based task completion time is measured}

\section{From right(camera) view, right-arm based task completion time is measured}

Fig. 8 Pose estimation using two camera angles to assess right and left arm task completion times.

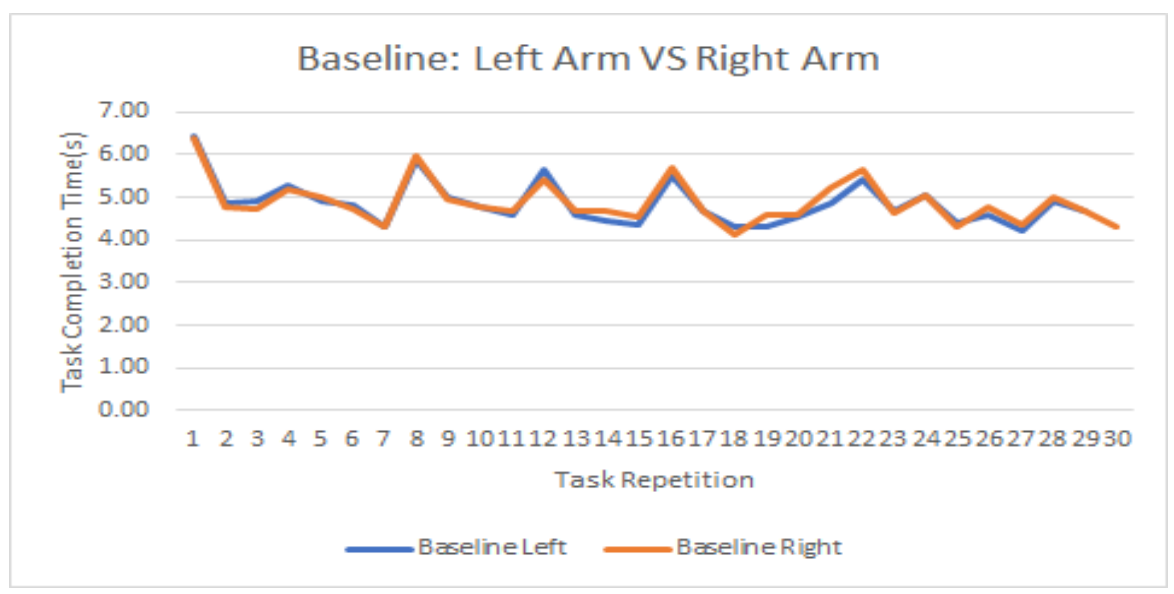

(a)

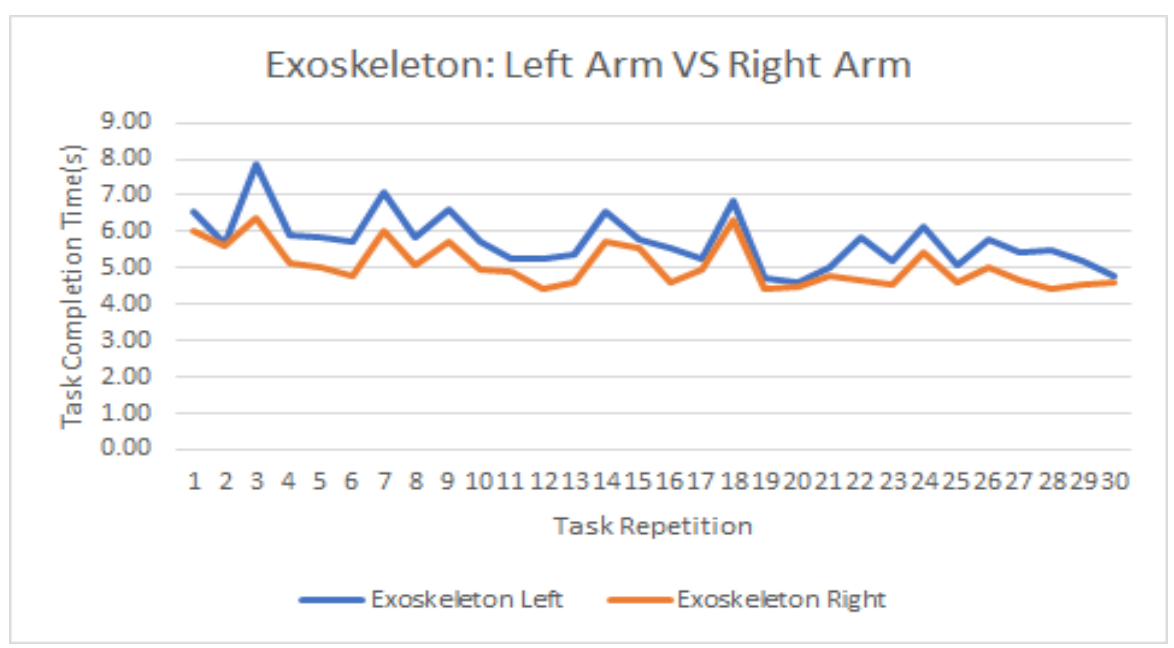

(b)

Fig. 9 Intra-subject wrist height profile for the left and right arm without support (a) and with exoskeleton support (b). 


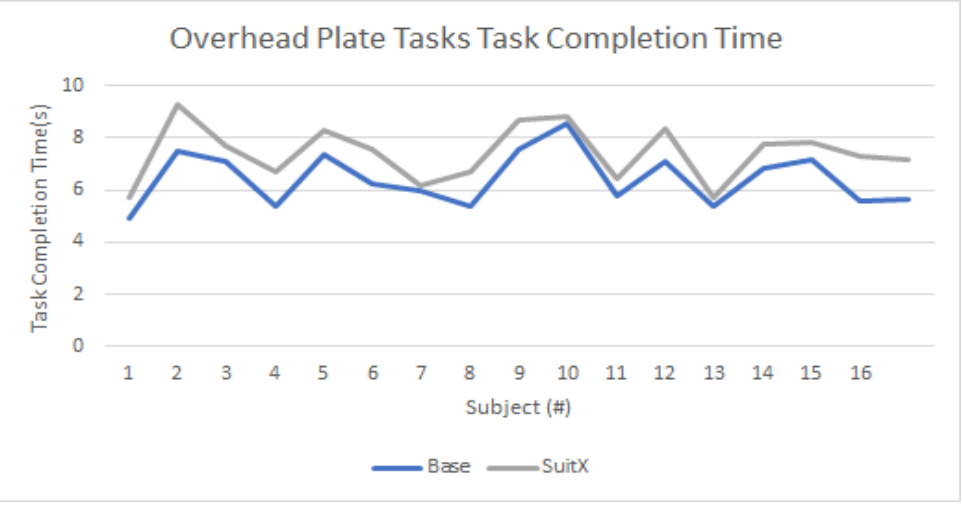

(a)

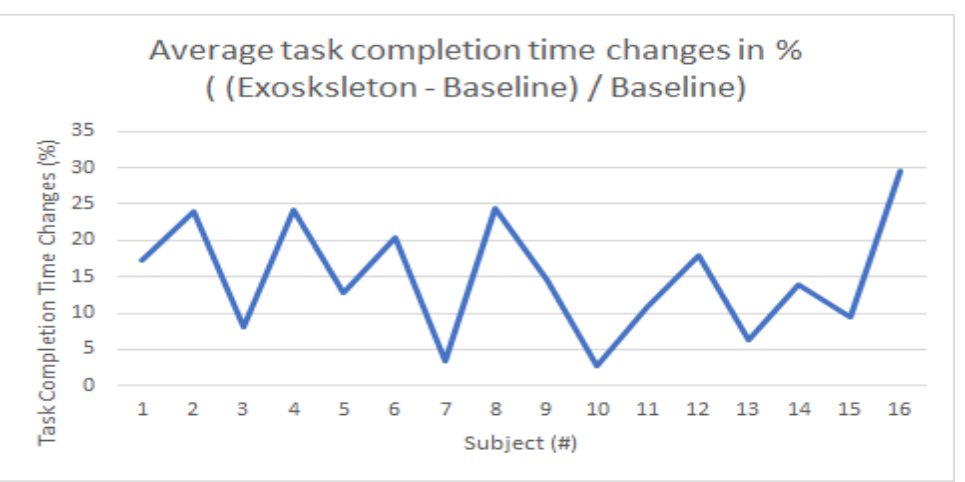

(b)

Fig. 6 Inter-subject summary statistics of the (a) task completion time between the test done without support and with exoskeleton support and (b) the time differential in \% between the two tests for each subject.

\subsubsection{Perceived Task Exertion}

One of the objectives of the survey was to assess the degree of correlation between the perceived task exertion and the physiological measurement (heart rate) with the goal of understanding how well the survey questions correlate to physiological measurements. As physiological measurements provide insights into the total body exertion, we assessed whether there was a statistical relationship between the heart rate and subject's perception of whether the task reached their maximum physical intensity, as shown in Fig. 11 and Table 6. 


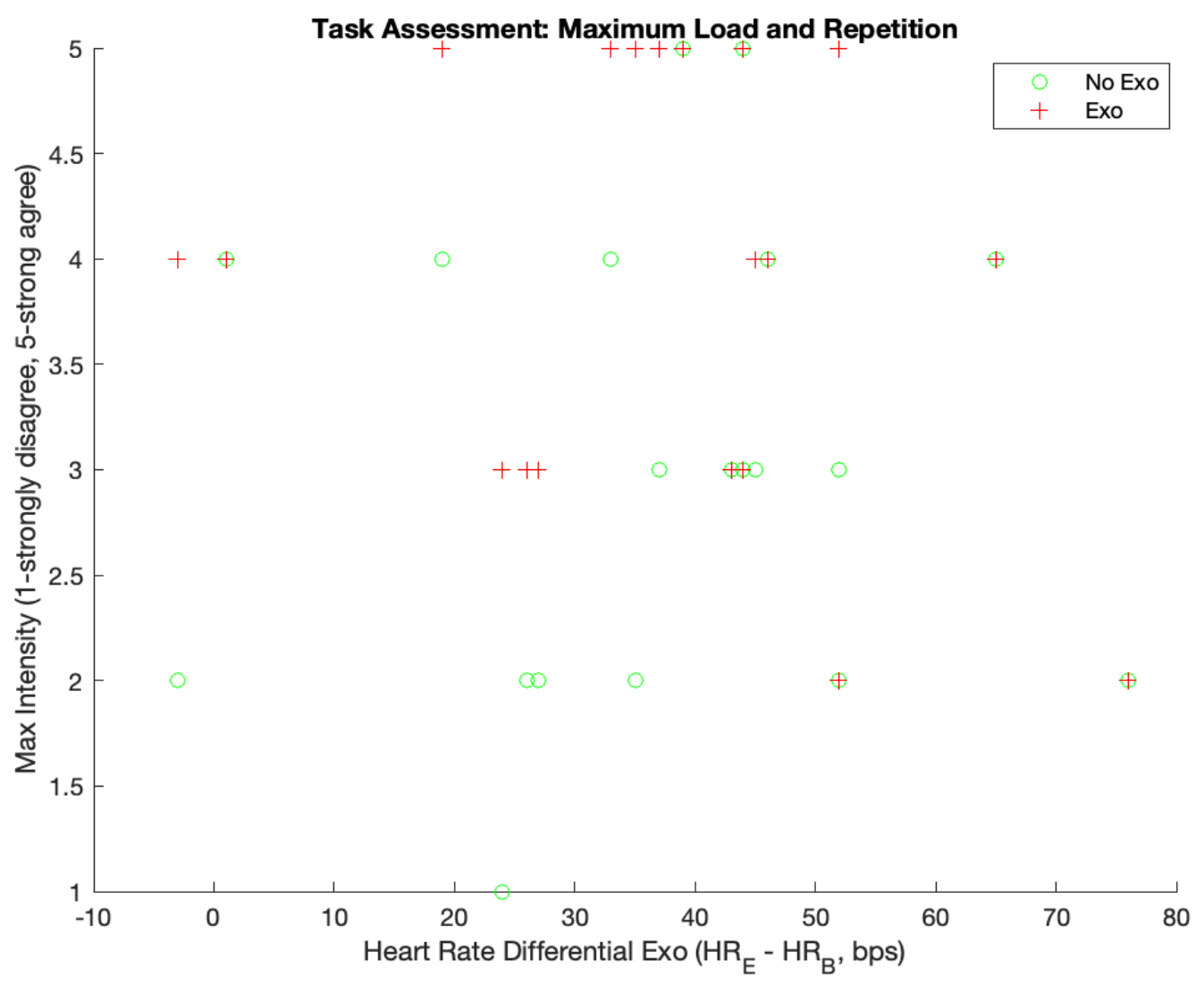

Fig. 11 Scatter plot of relationship between heart rate and the survey question on maximum load and repetition.

The rank correlation coefficient, $r$, obtained between the maximum intensity without an exoskeleton and the differential heart rate without an exoskeleton exceeded the critical value of 0.309 for a sample size of $N=19$ at the $90 \%$ confidence level, indicating a statistically significant association. On the other hand, regarding the question whether the task achieved the maximum load while wearing the exoskeleton, the association was not statistically significant $r<0.309$. Discussions on the possible interpretations and type of scale and associated description for future peg-in-hole tests are described in Section 4.1. The physical exertion was primarily attributed to musculoskeletal strain. In this case, using a method to measure musculoskeletal strain, such as EMG sensors may be a better indicator of task intensity than heart rate for the peg-in-hole test.

Although an EMG was not instrumented during the test, a possible indicator of muscle strain is perceived soreness/discomfort. The relationship of the perceived soreness or discomfort during the test to the ending heart rate without the exoskeleton was 0.68 . This seems to indicate that as the soreness increased, the heart rate also increased. The correlation between soreness/discomfort without the exoskeleton was highest for the ending heart rate, potentially indicating repetitive strain. With the exoskeleton, the correlation between

soreness/discomfort was the highest after 15 repetitions, rather than at the end, where the user may have benefited more from the exoskeleton after learning to coordinate with the 
exoskeleton's mechanical interface. As the user learned to work with the exoskeleton, the ending heart rate decreased.

The perceived intensity of the task without the exoskeleton had a significant correlation, above the $95 \%$ confidence interval, with the perceived usefulness of the exoskeleton. The result may indicate that the exoskeleton was perceived to be more useful when the without exoskeleton task was considered to be more strenuous. Tasks that were not perceived to be strenuous to the user, limited the user perception of the usefulness of the exoskeleton.

Table 6 Task Heart rate and correlation with exertion survey questions (correlation coefficient, $\boldsymbol{r}$ ).

\begin{tabular}{|c|c|c|c|}
\hline & $\begin{array}{l}\text { Without } \\
\text { exoskeleton }\end{array}$ & Exoskeleton & $\begin{array}{l}\text { Without exoskeleton - } \\
\text { Exoskeleton }\end{array}$ \\
\hline Heart Rate (differential) v. Task Intensity & -0.31 & -0.24 & -0.35 \\
\hline $\begin{array}{l}\text { Exoskeleton Usefulness v. Task Intensity (Without } \\
\text { exoskeleton) }\end{array}$ & 0.50 & 0.44 & -0.12 \\
\hline Heart Rate (end) v. Soreness/Discomfort & 0.68 & 0.43 & N/A \\
\hline Heart Rate (after 15 reps) v. Soreness/Discomfort & 0.49 & 0.60 & $\mathrm{~N} / \mathrm{A}$ \\
\hline
\end{tabular}

\section{Discussion}

Test methods are needed to assess the safety of and understand the human interactions with and task suitability for exoskeleton use. This study evaluated one potential peg-in-hole test method on the perceived comfort and strain as well as on quantitative measures on physiological strain in terms of heart rate, and finally on productivity with respect to task rate. The test method included three different task angle ranges for each subject, with exact angle ranges dependent on the test subject's technique and height. The task angle ranges included overhead, wall, and floor. Each component of the task was intended to demonstrate how the PoLoTAE can be applied and to utilize the different components of the full exoskeleton, which was comprised of shoulder, back, and leg supports. The test was intended to assess the mobility, productivity, comfort, and strain/fatigue of the user in the exoskeleton when adapting to a task that requires multiple task angles.

Ten of the 19 subjects found the floor pegs were the easiest to complete with the exoskeleton, and the exoskeleton provided the most beneficial support for the hips, legs, and knees. The results were generally consistent with another study on user perception of comfort for lowerlimb exoskeleton support [15].

However, even if the use of an exoskeleton provides limited user productivity, the ability to sustain users in supporting the completion of tasks without injuries can provide long term productivity and economic savings due to reduced injuries, the ability to work for longer durations, and worker retention.

Potential considerations for future test method development include:

- Where feasible, tailoring the task to the optimal range of angles of the exoskeleton's intended use would be helpful to fully utilize the exoskeleton's capabilities. This can be used by modifying the height of the task to the user. Our test focused on a single height since many jobs may not be able to be tailored to the worker's height.

- Where feasible, design tests that are sufficiently strenuous for the test subject to better utilize the exoskeleton's capabilities. 
- Include procedures to tune the parameters of the exoskeleton to a user's preference relative to the task.

- Time permitting, a training and fitting period for the subject to become accustomed to and to use the exoskeleton comfortably in order to minimize contact pressure may help with user perception of the exoskeleton.

- Time permitting, collect anthropometric (tailor) measurements to support exoskeleton fit.

- Include additional sensors, such as contact pressure, floor pressure, and force plates to assess the test method characteristics and safety relative to each subject, including: (a) the kinetic profile of the user and the exoskeleton (b) the balance and slippage risk profile, (c) the physiological intensity profile of the task, and (d) the posture profile while the user completes the tasks.

- The video-based pose estimation and tracking were limited to the overhead tasks due to occlusions. Further study on stereophotogrammetry methods and the associated systematic, random, and external errors and error propagation, such as camera placement, marker movement artifacts, pose estimation algorithmic improvements, repeatability, and reproducibility analysis are needed to have a reliable measurement method for evaluating human-exoskeleton task performance and biomechanical features.

There is also a need to be able to evaluate exoskeletons in the field to fully understand the worker demographics, expertise, and to retain the fidelity of the task characteristics the exoskeleton was intended for [8].

\subsection{Future Tests}

\subsubsection{Task}

The main suggestion to improving the task fidelity and to fully utilize the exoskeleton, was to increase the repetitions and duration to better leverage the exoskeleton's intended application. During a subsequent tour of an automotive manufacturing facility, the study team inquired and observed the typical task durations and worker-to-task positions to improve future tests. To evaluate an exoskeleton, it is useful to understand the intended task profile, including task variables such as the horizontal and vertical positions of the task's target destination (relative to the human or to the ground), the asymmetry of the task (twisting requirement), and the average and the maximum load of the object (tool). For tasks that only require a specific muscle group and worker-to-task angle range, it would be useful to separate the full peg-in-hole test to the specific task angle range. For example, if the task was undercarriage drilling at an automobile assembly plant, the PoLoTAE would be set at the specified task height with the typical number of repetitions or duration. The torque profile of the exoskeleton joint can also be adjusted to meet the user's preferences for the task. Additionally, a task angle profile of the intended activity for exoskeleton evaluation should be developed to increase the task simulation fidelity, and for fully assessing the exoskeleton 
capabilities with respect to the intended task. For the peg-in-hole test method, one user suggested a task that requires the user to hold the drill in the same general position for longer continuous periods and to exert a minimum force to increase the simulation fidelity of tightening a screw or bolt.

\subsubsection{Survey}

To align the user's perceived exertion to their level of fitness, one may consider using the Borg Rating of Perceived Exertion (RPE) scale, which is a relative scale [30]. The scale runs from 6 to 20 to align to the user's heart rate, where 6 is aligned with the resting heart rate. In previous tests, RPE was found to be correlated with heart rate at coefficients ranging from 0.50 to 0.70 . Another option is to increase the range and provide a scale from 1 to 10 .

Similarly, for the task exertion, the survey results indicated stronger association between the differential heart rate at the beginning and the end of the test and the question on maximum load while not wearing the exoskeleton. However, the correlation between the differential heart rate while wearing the exoskeleton and the perceived survey question on maximum intensity with the exoskeleton was lower. One interpretation for the weaker association while wearing the exoskeleton is the increased cognitive effort of learning and working with the exoskeleton while completing the task. The weight and heat of the exoskeleton can create a perception of being more arduous to interact with, though may not translate fully into additional cardiovascular burden. Another possibility is the need for additional measurements to understand the difference in muscle activity, that may cause the subject to perceive the exoskeleton to require greater effort.

\subsubsection{Additional measurements and metrics}

Use of kinetic sensors can provide quantifiable insights in how and how much musculoskeletal support the exoskeleton provides for the floor, wall, and overhead tasks in the test. Force plates or pressure sensors can also provide insights on whether the exoskeleton created additional imbalance or strain for the subject while alleviating other musculoskeletal strain in the shoulder, back or legs. Future work will include development of test and measurement methods to assess (1) the impact of exoskeletons on transferring joint force and pressure on to other areas of the body; (2) task precision; and (3) error propagation in the kinematic and kinetic measurement methods.

\section{Conclusion}

The peg-in-hole exoskeleton study evaluated a proposed test method to assess the safety, comfort, usability, and productivity impacts of an exoskeleton with three sets of support profiles, from overhead work to floor work, in order to evaluate multiple industrial peg-inhole task scenarios. The full set of tasks also allowed the researchers and users to understand how each exoskeleton support profile can be used and to design the test method to maximize the benefits of industrial exoskeletons. The study demonstrated how the PoLoTAE can be used to simulate typical industrial overhead tasks, where the test subjects agreed the industrial task platform provided a high-fidelity simulation.

The study helped to assess the capabilities and limitations of the test procedures and measurement methods to improve evaluation of a variety of exoskeletons with a full range of 
support profiles. The first measurement objective in our exoskeleton tests was to minimize the number of sensors that may limit a subject's movement or cause distractions during the test, such as optical tracking markers, IMUs, or EMG sensors. However, we noted that the use of additional sensors may be useful to further assess whether the qualitative questions on comfort and work intensity provide meaningful indicators for future test methods. The second measurement objective is the use of low-cost sensors to allow the test method to be versatile in both laboratory and field settings. The test methods developed helped to inform the development of industrial exoskeleton standard test methods. The data acquired from these human subject studies can inform the development of human-exoskeleton models, model validation, and analysis methods of future exoskeleton standard test methods. 


\section{Acknowledgments}

The authors would like to thank the test subjects who performed the described experiments through the NIST exoskeleton research study, IRB EL-2018-0060, for their time and input.

Certain commercial equipment, instruments, or materials are identified in this technical note to foster understanding. Such identification does not imply recommendation or endorsement by the National Institute of Standards and Technology, nor does it imply that the materials or equipment identified are necessarily the best available for the purpose.

\section{References}

[1] Bureau of Labor Statistics (2020) Fact Sheet $\mid$ Occupational injuries and illnesses resulting in musculoskeletal disorders (MSDs). Available at https://www.bls.gov/iif/oshwc/case/msds.htm.

[2] National Research Council and the Institute of Medicine (2001) Musculoskeletal disorders and the workplace: Low back and upper extremities. (National Academy Press, Washington D.C.).

[3] De Looze M, Bosch T, Krause F., Stadler KS, and O'Sullivan LW (2016) Exoskeletons for industrial application and their potential effects on physical workload. Ergonomics, 59(5): 671-681.

[4] Bostelman R, Messina E, and Foufou S (2017) Cross-industry standard test method developments: from manufacturing to wearable robots. Frontiers of Information Technology \& Electronic Engineering, 18(10): 1447-1457.

[5] Bostelman R and Hong T (2018) Test methods for exoskeletons-lessons learned from industrial and response robotics. Wearable exoskeleton systems: design, control and applications, (13): 335-361.

[6] ASTM International (2020) ASTM F3323 - Standard Terminology for Exoskeletons and Exosuits (ASTM International, West Conshohocken, PA). https://doi.org/10.1520/F3323-20

[7] Harvard Biodesign Lab (2021) Soft Exosuits. Available at https://biodesign.seas.harvard.edu/soft-exosuits.

[8] Nussbaum MA, Lowe BD, de Looze M, Harris-Adamson C, and Smets M (2019) An Introduction to the Special Issue on Occupational Exoskeletons. IISE Transactions on Occupational Ergonomics and Human Factors, 7(3/4):153-162.

[9] International Organization for Standardization (ISO) 13482 (2014) Robots and robotic devices -- Safety requirements for personal care robots, https://www.iso.org/standard/53820.html.

[10] Maxheimer E, Shanahan C, Wolf A, Craig J, Mountjoy D, Johnson C, and Canzonetta D (2019) A human systems integration approach to industrial exoskeleton evaluations for the USAF. 711th Human Performance Wing Wright-Patterson AFB United States, Tech. Rep.

[11] Crowell HP, Park, JH, Haynes CA, Neugebauer JM, and Boynton AC (2019) Design, evaluation, and research challenges relevant to exoskeletons and exosuits: A 26-year perspective from the U.S. Army Research Laboratory. IISE Transactions on Occupational Ergonomics and Human Factors, 7(3/4): 199212.

[12] Van Engelhoven L, Poon N, Kazerooni H, Rempel D, Barr A, and Harris-Adamson C (2019) Experimental evaluation of a shoulder-support exoskeleton for overhead work: Influences of peak torque amplitude, task, and tool mass. IISE Transactions on Occupational Ergonomics and Human Factors, 7(3/4):250-263.

[13] Bostelman R, Virts A, Shah M, Yoon S, and Li-Baboud Y (2020) Development of a Kinematic Measurement Method for Knee Exoskeleton Fit to a User. (National Institute of Standards and 
Technology, Gaithersburg, MD), NIST Technical Note (NIST TN) 2107.

https://doi.org/10.6028/NIST.TN.2107.

[14]Huysamen K, de Looze M, Bosch T, Ortiz J, Toxiri, S, and O'Sullivan LW (2018) Assessment of an active industrial exoskeleton to aid dynamic lifting and lowering manual handling tasks. Applied Ergonomics, 68:125-131.

[15]ASTM International Committee F48 on Exoskeletons and Exosuits (2021). Available at https://www.astm.org/committee-f48

[16]Bostelman R, Li-Baboud Y, Virts A, Yoon S, and Shah M (2019) Towards Standard Exoskeleton Test Methods for Load Handling. In 2019 Wearable Robotics Association Conference (WearRAcon), (IEEE, Scottsdale, AZ), pp. 21-27.

[17]De Rossi SM, Vitiello N, Lenzi, T, Ronsse R, Koopman B, Persichetti A, Vecchi F, Ijspeert AJ, Van der Kooij H, and Carrozza MC (2011) Sensing pressure distribution on a lower-limb exoskeleton physical human-machine interface. Sensors, 11(1):207-227.

[18]Jang J, Kim K, Lee J, Lim B, and Shim Y (2015) Online gait task recognition algorithm for hip exoskeleton. In 2015 IEEE/RSJ International Conference on Intelligent Robots and Systems (IROS), (IEEE, Hamburg, Germany), pp. 5327-5332.

[19] Wheeler J, Rohrer B, Kholwadwala D, Buerger S, Givler R, Neely J, Hobart C, and Galambos P (2006) Insole MEMs pressure sensing for a lower extremity exoskeleton. The First IEEE/RAS-EMBS International Conference on Biomedical Robotics and Biomechatronics (BioRob), (IEEE, Pisa, Italy), pp. 31-34.

[20] Spada S, Ghibaudo L, Gilotta S, Gastaldi L, and Cavatorta MP (2017) Analysis of exoskeleton introduction in industrial reality: main issues and EAWS risk assessment. In International Conference on Applied Human Factors and Ergonomics (Springer, Cham, Los Angeles, CA), pp. 236-244.

[21] Marras WS (1990) Industrial electromyography (EMG). International Journal of Industrial Ergonomics, 6(1):89-93.

[22] Weston EB, Alizadeh M, Knapik GG, Wang X, and Marras WS (2018) Biomechanical evaluation of exoskeleton use on loading of the lumbar spine. Applied ergonomics, 68:101-108.

[23] Bär M, Steinhilber B, Rieger MA, and Luger T (2021) The influence of using exoskeletons during occupational tasks on acute physical stress and strain compared to no exoskeleton-A systematic review and meta-analysis. Applied Ergonomics, 94:103385.

[24]Zhang W, White M, Zahabi M, Winslow AT, Zhang F, Huang H, and Kaber D (2016) Cognitive workload in conventional direct control vs. pattern recognition control of an upper-limb prosthesis. IEEE International Conference on Systems, Man, and Cybernetics (SMC) (IEEE, Budapest, Hungary), pp. 23352340 .

[25]Kirkwood GL, Otmar CD, and Hansia M (2021) Who's Leading This Dance?: Theorizing Automatic and Strategic Synchrony in Human-Exoskeleton Interactions. Frontiers in Psychology, 12.

[26] SuitX (2021) Occupational Exoskeletons: An Introduction. Available at https://www.suitx.com/introduction

[27] Cao Z, Hidalgo G, Simon T, Wei SE, and Sheikh Y (2019) OpenPose: realtime multi-person 2D pose estimation using Part Affinity Fields. IEEE Transactions on Pattern Analysis and Machine Intelligence, 43(1):172-186.

[28] Ramsey PH (1989) Critical values for Spearman's rank order correlation. Journal of educational statistics, 14(3):245-253. 
[29] Gillette JC and Stephenson ML (2019) Electromyographic assessment of a shoulder support exoskeleton during on-site job tasks. IISE Transactions on Occupational Ergonomics and Human Factors, 7(3-4): 302310 .

[30]Borg GA (1998) Borg's Perceived Exertion and Pain Scales. Human Kinetics.

[31]Lu TW and Chang CF (2012) Biomechanics of human movement and its clinical applications. Kaohsiung Journal of Medical Sciences, 28:S13-25.

[32] A. Burdorf, G. Govaert and L. Elders. Postural load and back pain of workers in the manufacturing of prefabricated concrete elements. Ergonomics, 34 (1991), pp. 909-918

[33] Luger T, Cobb TJ, Seibt R, Rieger MA, and Steinhilber B (2019) Subjective Evaluation of a Passive Lower-Limb Industrial Exoskeleton Used During Simulated Assembly, IISE Transactions on Occupational Ergonomics and Human Factors, 7(3/4):175-184.

[34]Butler T and Gillette JC (2019) Exoskeletons: Used as PPE for Injury Prevention. Professional Safety, pp. 33-37.

[35] Schmalz T, Schändlinger J, Schuler M, Bornmann J, Schirrmeister B, Kannenberg A, Ernst M (2019) Biomechanical and Metabolic Effectiveness of an Industrial Exoskeleton for Overhead Work. International Journal of Environmental Research and Public Health, 16(23):4792.

[36] Gillette J (2020) Lab and field studies to assess if exoskeleton usage reduces muscle fatigue risk. ErgoX Symposium, (Human Factors and Ergonomic Society), p.9.

[37]Kim S, Nussbaum MA, Esfahani MI, Alemi MM, Alabdulkarim S, Rashedi E (2018) Assessing the influence of a passive, upper extremity exoskeletal vest for tasks requiring arm elevation: Part I"Expected" effects on discomfort, shoulder muscle activity, and work task performance. Applied ergonomics, (70):315-322. 